Br Heart J 1987;58:441-6

\title{
Review
}

\section{Ventricular defibrillation: the Belfast experience}

\author{
G W N DALZELL, S R CUNNINGHAM, C M WILSON, J D ALLEN, * \\ J ANDERSON, $\uparrow$ A A J ADGEY
}

From the Regional Medical Cardiology Centre, Royal Victoria Hospital and the ${ }^{\star}$ Department of Physiology, Queen's University, Belfast and $\uparrow$ Bioengineering Department, University of Ulster, Jordanstown, Northern Ireland

The single greatest cause of death in these islands is coronary artery disease. Over $60 \%$ of premature deaths from acute myocardial infarction occur within the first hour of the onset of symptoms and are thus termed "sudden". Ventricular fibrillation is the cause of sudden death in over $90 \%$ of patients with myocardial infarction who usually collapse at home. Rapid detection and correction are major determinants of the outcome of ventricular fibrillation.

\section{Correction of ventricular fibrillation}

Ventricular fibrillation requires a focus to initiate the disordered ventricular activity, a critical mass to maintain the fractionated activity, and a critical relation between the conduction velocity and the refractory period to produce microreentry movements. Although most patients with ventricular fibrillation require DC shock to correct ventricular fibrillation, occasionally ventricular fibrillation is self limited or can be corrected by a chest thump. Before cardiopulmonary resuscitation is started the lower sternum should be thumped with the fist when the patient is lying on a firm surface.

Because defibrillators are not usually immediately available at the scene of collapse, lay people should be trained to maintain efficient cardiopulmonary resuscitation until a defibrillator arrives. Regular retraining is essential to meet these demanding requirements and also because an operator is unlikely to have to deal with more than one or two collapses during their lifetime.

The first DC defibrillator systems were mains powered but small battery operated systems were developed and these now weigh less than $7 \mathrm{lbs}(3 \mathrm{~kg})$.

The optimal discharge from a defibrillator is the one which requires the lowest voltage, current, and

Requests for reprints to Dr A A J Adgey, Regional Medical Cardiology Centre, Royal Victoria Hospital, Belfast BT12 6BA.

Accepted for publication 7 July 1987 delivered electrical energy. At present damped sine waves and trapezoid and square waveforms are used in commercial defibrillators but we do not know which of these waveforms or combinations are most successful in correcting ventricular fibrillation and which cause the least myocardial damage. Limitation of this damage is an additional reason why it is important to achieve successful defibrillation with the first shock:

\section{Paddle positions}

If the paddles are incorrectly positioned on the chest wall successful defibrillation is unlikely. One paddle should be placed to the right of the sternum immediately under the clavicle and the other over the region of the apex of the left ventricle. Because at discharge the current density is greatest at the edge of the paddle the application site appears almost immediately as erythematous rings in the skin which usually persist for at least $24-48$ hours.

\section{Disposable electrocardiogram/defibrillator pads}

The use of a combined oscilloscope and defibrillator with disposable pads has shortened the interval between cardiac arrest and defibrillation. When the pads are placed at the base of the heart and over the apex the electrocardiogram is immediately displayed. There is no need to attach electrocardiogram electrodes to the chest wall or to the arms or legs to determine the rhythm on an oscilloscope before attempting defibrillation with hand held paddles. Correct positioning of the pads is crucial to both analysis and defibrillation.

The optimal size of pads and their content have not yet been determined. We have examined transthoracic impedance and electrode pad size in attempted defibrillation during cardiac arrests from ventricular fibrillation and ventricular tachycardia. 
Transthoracic impedance was measured before countershock by passing a $30 \mathrm{kHz}$ current through the chest via self-adhesive electrocardiogram/ defibrillator pads (R2) applied anteroanteriorly. Pad diameters were $8 \mathrm{~cm} / 8 \mathrm{~cm}$ (small) and $8 \mathrm{~cm} / 12 \mathrm{~cm}$ (intermediate), and $12 \mathrm{~cm} / 12 \mathrm{~cm}$ (large). The first two shocks were of $200 \mathrm{~J}$ (delivered energy) and subsequently $360 \mathrm{~J}$ shocks were used. Mean (SD) transthoracic impedance was higher with the small pads $(112.7(24) \Omega)$ than with the intermediate $(90.4$ $(23) \Omega)$ or large ones $(66.8(12) \Omega)(p<0.0005)$. Two shocks of $200 \mathrm{~J}$ were successful in all 23 patients treated with large pads, 78/104 (75\%) patients treated with intermediate pads, but in only $23 / 42$ $(55 \%)$ patients treated with small pads $(p<0.01)$. Successful cardioversion of primary ventricular fibrillation by a single $200 \mathrm{~J}$ shock was more often achieved with large pads than with intermediate or small pads $(83 \%, 63 \%, 31 \%$ respectively). Because transthoracic impedance is higher with small pads the success of defibrillation is reduced. The greatest success and lowest transthoracic impedance were obtained with $12 \mathrm{~cm} / 12 \mathrm{~cm}$ pads.

\section{Energy levels for defibrillation}

Eighty five per cent of episodes of ventricular fibrillation were corrected by a single shock of 200 W.s stored energy irrespective of the time the patient was in ventricular fibrillation or the patient's weight. ${ }^{1}$ In a further $9 \%$ a second $200 \mathrm{~W}$.s shock was successful. A single $100 \mathrm{~W}$.s stored energy shock was successful in $67 \% .^{1}$ Correction by a single $100 \mathrm{~W} . \mathrm{s}$ shock was more likely the shorter the time the patient had been in ventricular fibrillation. Thus energies of less than $400 \mathrm{~W} . s$ stored should be used initially to correct ventricular fibrillation.

\section{Number of shocks to correct ventricular fibrillation}

At the initial cardiac arrest $41 \%$ of patients with ventricular fibrillation within the first hour of myocardial infarction required $\leqslant 2$ shocks for cardioversion. ${ }^{2}$ Some required three and some four; very few required $>10$ shocks. Patients with ischaemic heart disease who had ventricular fibrillation within 12 hours of the onset of symptoms required an average of two shocks to correct ventricular fibrillation. ${ }^{3}$ This information helps to reduce the size and weight of defibrillators because the size of the capacitor in the defibrillator is determined by the number of shocks to be delivered.

The number of shocks that a patient requires to correct ventricular fibrillation is relevant to their long term survival. In 1986 we looked at 125 patients with ventricular fibrillation. ${ }^{4}$ Most patients with a first arrest who required either one or two shocks to correct ventricular fibrillation survived to leave hospital whereas the death rate was significantly higher in those who needed more than three shocks. Discriminant function analysis of the factors measured at the time of the initial arrest in these 125 patients was used to identify those of greatest significance in predicting survival to leave hospital. The most significant factor was the application of five shocks or less to correct ventricular fibrillation. This was more important than the time from onset of ventricular fibrillation to the first shock, the presence of primary ventricular fibrillation (ventricular fibrillation in the absence of heart failure or cardiogenic shock), or the absence of previous antiarrhythmic treatment.

\section{Prognosis of patients in ventricular fibrillation}

If ventricular fibrillation occurs early after the onset of symptoms of acute myocardial infarction it is associated with an excellent long term prognosis after successful resuscitation. When ventricular fibrillation occurred more than four hours after the onset of symptoms (these were mainly patients with acute myocardial infarction), approximately $80 \%$ were alive three years after the initial episode. ${ }^{5} \mathrm{~A}$ similar outcome was found in patients with myocardial infarction uncomplicated by ventricular fibrillation. When ventricular fibrillation occurs $\geqslant 4$ hours after the onset of symptoms the prognosis is poorer, mainly because of the extent of muscle damage. In those who have ventricular fibrillation complicating ischaemic heart disease without an acute myocardial infarction, ventricular fibrillation tends to recur and long term antiarrhythmic agents, with or without cardiac surgery, have been used in an attempt to reduce the recurrence rate of ventricular fibrillation. Automatic implantable cardioverters or semiautomatic or automatic external defibrillators can be used for the recurrence of ventricular fibrillation in these patients.

\section{Damage during defibrillation}

Although defibrillation is a life saving procedurewhen cardiac arrest is caused by ventricular fibrillation or ventricular tachycardia there is no established alternative-we should not ignore its potential to cause injury. The prognosis of patients who receive multiple shocks remains poor and it is difficult to measure the adverse effect of shocks under clinical conditions.

The effects of 1,5 , or 10 synchronised ( $400 \mathrm{~W} . \mathrm{s}$ 
stored) transthoracic shocks delivered at 30 second intervals to adult greyhounds were studied in the laboratory. ${ }^{67}$ With an increase in the number of shocks more dogs died acutely from either asystole or electromechanical dissociation (one shock, $0 / 6$ (there was one late death in the single shock group, probably from respiratory depression); five shocks, 8/18; 10 shocks, 12/17). Electron microscopy showed few abnormal myocardial features when compared with dogs that had been anaesthetised and ventilated but had not received any shocks. ${ }^{8}$ In particular the myofibrillar structure remained intact and there was no disruption of the cell membrane or intercalated discs. Clumping of the nuclear chromatin was common in the myocardium of the shocked animals, although we do not have enough evidence to say how specific this feature is. The lack of a structural abnormality suggests that the basic defect at this early stage is at molecular or ionic level.

In the animals surviving five or ten shocks important ST segment elevation was recorded from the right and left precordial leads 15 minutes and one hour after the shocks. This had resolved by 72 hours. In the group that received one shock the ST segment did not change. Electrocardiogram rhythm strips recorded 24, 48, and 72 hours after the shocks showed sustained ventricular tachycardia as the dominant cardiac rhythm at 24 hours after five or ten shocks, with no difference in the ventricular ectopic count per minute in the two groups.

The macroscopic cardiac damage three days after the shocks was greater in the 10 shock group than in the five shock group. A single shock caused minimal damage in one out of five dogs in this group; the four other hearts were undamaged.

The influence of defibrillator waveform on cardiac damage has also been studied. The defibrillation threshold for various waveforms was first established. ${ }^{89}$ For a series of trapezoidal waveforms lasting 2.5-55 ms the threshold current fell with increasing pulse duration up to $20 \mathrm{~ms}$ but was increased at 40 and $55 \mathrm{~ms}$. The threshold delivered energy was not different for pulses of $2.5-20 \mathrm{~ms}$ but for the 40 and $55 \mathrm{~ms}$ pulses it was significantly higher. The defibrillation threshold of the 5 and 20 ms trapezoid waveforms was compared with that of the three clinically available types of damped sine waves (Belfast, Edmark, and Lown). The threshold current of the $5 \mathrm{~ms}$ trapezoid and the three damped sine waves was not significantly different. Although the $20 \mathrm{~ms}$ trapezoid waveform had a threshold current that was significantly lower than that of Belfast and Edmark waveforms, its delivered energy was significantly greater than that of the four other waveforms. Thus at the threshold level of defibrillation no one of these waveforms was superior.
A further group of dogs was randomised to receive five synchronised shocks from one of these five waveforms (each shock: $400 \mathrm{~J}$ stored energy for the three sinewaves; $1100 \mathrm{~V}$ on capacitor for trapezoidal waveforms). ${ }^{8-10}$ The ventricular ectopic activity recorded at 24 and 48 hours was not significantly different in the groups receiving the $20 \mathrm{~ms}$ trapezoid and three damped sine waves. Little ectopic activity was recorded in the $5 \mathrm{~ms}$ trapezoid group. The ST segment elevation $15 \mathrm{~min}$ after the shocks was significantly greater in the Belfast and Edmark groups than in the Lown and $20 \mathrm{~ms}$ trapezoidal groups. The cardiac damage at three days was significantly greater in the Belfast and Edmark groups than in the Lown group. The long $(20 \mathrm{~ms})$ trapezoidal wave caused significantly more damage than the shorter trapezoidal wave. Although the Lown wave caused less injury than the two other sine waves, it also had the lowest delivered energy. A comparison of the peak current shows that the Edmark defibrillator generated the highest peak transthoracic current. The current and energy are influenced by the total circuit resistance, which is made up of the internal defibrillator resistance and transthoracic impedance of the dog, which is generally lower than the average values reported for man. Added to this the degree of damping of the waveforms may also have influenced the damage caused.

These animal studies have shown that the heart is damaged by defibrillating shocks and that the available sine waves and a number of trapezoidal waves all easily achieve defibrillation at threshold level. Information on the current required for successful human defibrillation is limited. Knowledge of this factor would help in the evaluation of different waveforms both in the laboratory and clinical setting. If the transthoracic current required for human defibrillation is established, it may become possible to construct trapezoidal defibrillators that can be compared with the damped sine wave defibrillators under clinical conditions.

\section{Peak current at defibrillation}

Peak current at the time of defibrillation is probably more important in terms of successful defibrillation than energy and it depends on the patient's transthoracic impedance: This is why it is essential that predicted transthoracic impedance, which is assessed before the shock being passed across the chest, correlates well with the actual transthoracic impedance. Predicted transthoracic impedance was measured using a $30 \mathrm{kHz}$ pulse passed through the chest. Peak current at each discharge. was recorded. The actual transthoracic impedance was derived from calibration curves (peak current vs known 
resistances). The actual and predicted transthoracic impedances correlated well. The optimum peak current for defibrillation of the adult human in ventricular fibrillation appears to lie between 25 and $32 \mathrm{~A}$. We are investigating the use of such a system to increase the success rate of first shock defibrillation.

\section{Automatic and semiautomatic defibrillators}

Paramedical personnel have had extensive training in defibrillation and general practitioners can defibrillate patients successfully outside hospital. ${ }^{11} 12$ Nevertheless, because most cardiac arrests occur at home and the ultimate outcome is dependent on early defibrillation, the need for an automatic defibrillator for use in such circumstances by lay personnel has encouraged the development of systems to sense the presence of ventricular fibrillation. ${ }^{13-17}$ We have evaluated a microprocessor-based system that detects ventricular fibrillation and have determined its accuracy during the management of cardiac arrests. ${ }^{13}$ The development of a semiautomatic or automatic defibrillator depends on the results of such studies.

The initial algorithm for ventricular fibrillation detection used the following criteria: $(a)$ the zero content, that is the isoelectric segment is absent or very small; $(b)$ the energy density pattern is irregular; (c) slope irregularity; and $(d)$ heart rate variability detector. The success rate for the detection of the initial rhythm showed a sensitivity of $93 \%$ and a specificity of $97 \%$. Continuous analysis (every 8-18 seconds) of the electrocardiogram gave a sensitivity of $74 \%$ and for non-ventricular fibrillation sequences the specificity was $99 \%$. The unit has been updated with a ten bit input converter giving a total dynamic range from $0.2 \mathrm{mV}$ to $4 \mathrm{mV}$, and now the following two criteria have to be satisfied independently: ( $a$ ) absence of isoelectric segment; $(b)$ QRS width, variability, and regularity. The zero content is optimised by input filtering. If both $(a)$ and $(b)$ are met over two consecutive analyses, that is eight seconds, ventricular fibrillation is detected. If neither is met non-ventricular fibrillation is reported. If only $(a)$ or $(b)$ is satisfied for ventricular fibrillation, another four seconds trace is analysed. If still only one criterion is satisfied the system will reset and begin analysis again. Excessive artefact produced by cardiopulmonary resuscitation causes resetting of the system each second. The result is displayed visually every 8-12 seconds as nonventricular fibrillation or ventricular fibrillation. The electrocardiogram and its analysis are recorded simultaneously on tape. Later the analysis is compared with the electrocardiogram. When this system was used with continuous electrocardiogram analysis the sensitivity for ventricular fibrillation was $94 \%$ and the specificity for non-ventricular fibrillation was $97.5 \%$. Ventricular fibrillation of low amplitude $(<0.5 \mathrm{mV})$, cardiopulmonary resuscitation, or change in rhythm to ventricular fibrillation during the 8-12 $\mathrm{s}$ analysis period produced false negative results. False positive identification of ventricular fibrillation was caused by agonal rhythms and artefact induced by cardiopulmonary resuscitation.

\section{Improvements in the system for detecting ventricular fibrillation}

Increased sensitivity of the detection of rate variability has improved the identification of agonal rhythm. If the rhythm changes during analysis causing ventricular fibrillation to be incorrectly detected, then in a fully automatic system analysis will continue as the capacitor is charging. If at this stage ventricular fibrillation is not identified, the charge will be aborted and the analysis will continue.

A considerable number of patients who may require automatic defibrillation already have a pacemaker. The hardware is now designed to remove the effect of any pacemaker spikes on the analysis of ventricular fibrillation. The application of the DC countershock (particularly through pads that are also sensing the electrocardiogram) may cause several serious problems in an automatic system for the detection of ventricular fibrillation: $(a)$ electrode saturation, (b) amplifier saturation, (c) software corruption. When the signal to the defibrillator relay is about to be activated a parallel signal is supplied to the microprocessor. This signal (timed to operate the microprocessor before the defibrillator relay delivers the energy) switches the microprocessor to a "sleep mode". This mode stores the microprocessor's status registers in a non-corruptible form and stops the microprocessor operations. The "sleep mode" operates at $250 \mathrm{~m} / \mathrm{s}$, which is insignificant in terms of processing time but gives sufficient time for the defibrillator high voltage shock to be delivered. Then normal processing can begin again. This technique prevents corruption of the software during defibrillation.

There are broadly three types of unacceptable signals: (a) saturation potentials; (b) slowly changing baselines; (c) fast artefact (noisy baseline).

Saturation potentials are coped with by straightforward amplitude detection. When a certain limit is reached a fast acting integrator forces the baseline back to centre. In addition, the automatic analysis is stopped when saturation is detected and a "poor signal" indicator is switched on.

$A$ slowly changing baseline is handled by the low frequency break-point filter. If the $3 \mathrm{~dB}$ point is set 
at $2.5 \mathrm{~Hz}$ this condition does not cause any serious problems.

Fast rise signals (as in pacemaker spikes) are detected by the slope-detection portion of the algorithm. The slope detection not only provides a limit for ds/dt but also continues to reject the incoming signal if the turning points recur within a certain interval.

Activation of any of these artefact detectors stops processing and a signal indicates poor quality trace. Additional circuitry detects the high impedances caused by displacement of the electrodes.

Treatment of a cardiac arrest may require cardiopulmonary resuscitation and compression on the chest wall will generate substantial noise on the electrocardiogram signal. The effect of normal cardiopulmonary resuscitation on the algorithm is reduced by filtering the input signals.

\section{Operation of a semiautomatic/automatic external defibrillator}

Connection of the disposable electrocardiogram/ defibrillator electrodes to the patient's chest and raising the display panel activates the instrument. At all times the machine functions in the passive monitor mode with the first decision phase taking between 8-12 seconds. Any artefact detected during this period is registered as an unacceptable signal and processing is automatically stopped. If ventricular fibrillation is not detected at the first pass the unit returns to the monitor mode. If analysis indicates ventricular fibrillation an audio alarm asks the operator to confirm that the patient is unconscious. If the answer is "no" the unit again returns to automatic analysis. When it is "yes" the defibrillator begins to charge and as a final check simultaneous analysis continues. After $8 \mathrm{~s}$ the unit confirms ventricular fibrillation by displaying the instruction "shock" and telling the operator to stand away from the patient and activate a discharge action (semiautomatic).

Once the appropriate decision has been taken by the microprocessor it is a relatively simple matter to instruct the operator when to defibrillate (semiautomatic) or to signal the defibrillator to defibrillate (automatic). Again if ventricular fibrillation is not confirmed during a second phase of monitoring the machine will return to the original analysis mode.

Several other processes continue in parallel with the analysis and defibrillation modes. System diagnostics monitor the operation of hardware and software and the electrocardiogram is continually recorded on tape. A second track also records any appropriate voice messages from the operator. The system includes an electrocardiogram display with the appropriate message to the operator.

Semiautomatic and automatic systems are useful for training ambulance and paramedical staff. They enhance the confidence that these operators have in their diagnostic ability.

\section{Transtelephonic defibrillation}

Early defibrillation may be facilitated by electrocardiogram diagnosis and defibrillation by a physician who is connected by telephone to the patient or their spouse. The patient unit is an attache case containing a microprocessor, microphone, defibrillator, and electrocardiogram/defibrillator pads. The spouse applies the electrocardiogram/defibrillator pads to the patient's chest. The patient's unit is then connected to a standard telephone line and, once activated, automatically dials and activates the remotely sited base station, thus establishing twoway voice communication and electrocardiogram transmission. The physician operating the base station identifies the rhythm and controls the defibrillation (if required), charging, and discharge (with or without synchronisation). The positioning of the pads is assessed by transthoracic impedance monitoring at the base station. We have assessed the efficacy and safety of transtelephonic defibrillation in 19 patients (41-81 (mean $60 \mathrm{yr})$ ) undergoing 20 elective cardioversions. Eighteen had atrial fibrillation and one had ventricular tachycardia on two separate occasions. Time for activation of the base station by the patient unit ranged from 18 to 24 seconds (mean 20.5 seconds), and in all cardioversions clear voice communication and electrocardiogram signals were established. Sixty four DC shocks (50-360 J delivered) were administered safely and were successful in 18 of 20 cardioversions. All shocks were synchronised correctly and there were no adverse effects. The average discharge delay time from base station to patient unit was 2.6 seconds (range $1.5-5$ ).

We are on the threshold of many interesting developments based on biotechnology and communication. These will permit the operation of semiautomatic and automatic defibrillation systems in the home and should improve the opportunities for early defibrillation of patients at risk from ventricular fibrillation.

\section{References}

1 Campbell NPS, Webb SW, Adgey AAJ, Pantridge JF. Transthoracic ventricular defibrillation in adults. $\mathrm{Br}$ Med J 1977;ii:1379-81.

2 Logan KR, McIlwaine WJ, Adgey AAJ, Pantridge JF. Ventricular fibrillation and its recurrence in early acute myocardial infarction. Lancet $1981 ; \mathbf{i}: 242-4$. 
3 Logan. KR, McIlwaine WJ, Adgey AAJ, Pantridge JF. Recurrence of ventricular fibrillation in acute ischaemic heart disease. Circulation 1981;64:1163-7.

4 Dunn HM, McComb JM, MacKenzie G. Adgey AAJ. Survival to leave hospital from ventricular fibrillation. Am Heart J' 1986;112:745-52.

5 McNamee BT, Robinson TJ, Adgey AAJ, Scott ME, Geddes JS, Pantridge JF. Long-term prognosis following ventricular fibrillation in acute ischaemic heart disease. $\mathrm{Br}$ Med J 1970;iv:204-6.

6 Adgey AAJ, Allen JD, Wilson CM. Adverse effects of multiple DC countershocks on the canine heart [Abstract]. J Physiol (Lond) 1984;355:51P.

7 Wilson CM, Allen JD, Adgey AAJ. Death and damage after multiple DC countershocks [Abstract]. $\mathrm{Br}$ Heart $J$ 1985;53:99.

8 Wilson.CM, Allen JD, Bailey A, Anderson J, Adgey $\mathrm{AAJ}$. Is there an optimum waveform for cardiac defibrillation? [Abstract] X World Congress of Cardiology September 1986; p. 185.

9 Wilson CM, Bailey A, Anderson J, Allen JD, Adgey AAJ. Efficacy and safety of sine and trapezoidal defibrillator waveforms [Abstract]. Circulation 1985;72(suppl III):385.

10 Wilson CM, Bailey A, Anderson J, Allen JD, Adgey AAJ. Defibrillator waveform and cardiac injury [Abstract]. Br Heart J 1985;53:678.
11. Clyde C, Kerr A, Varghese A, Wilson C. Defibrillators in general practice. Br Med J 1984;289:1351.

12 Pai GR, Haites NE, Rawles JM. One thousand heart attacks in Grampian: the place of cardiopulmonary resuscitation in general practice. $\mathrm{Br}$ Med $J 1987$; 294:352-4.

13 Jack CM, Hunter EK, Pringle TH, Wilson JT, Anderson J, Adgey AAJ. An external automatic device to detect ventricular fibrillation. Eur Heart $J$ 1986;7:404-11.

14 Weaver WD, Copass MK, Hill DL, Fahrenbruch C, Hallstrom AP, Cobb LA. Cardiac arrest treated with a new automatic external defibrillator by out-ofhospital first responders. Am J Cardiol 1986;57: 1017-21.

15 Moore JE, Eisenberg MS, Andresen E, Cummins RO, Hallstrom A, Litwin P. Home placement of automatic external defibrillators among survivors of ventricular fibrillation. Ann Emerg Med 1986;15:811-2.

16 Stults KR, Brown DD, Kerber RE. Efficacy of an automated external defibrillator in the management of out-of-hospital cardiac arrest: validation of the diagnostic algorithm and initial clinical experience in a rural environment. Circulation 1986;73:701-9.

17 Cummins RO, Eisenberg MS, Stults KR. Automatic external defibrillators: clinical issues for cardiology. Circulation 1986;73:381-5. 
Our study was essentially a quantitative assessment of suppressor cell function, prompted by reports of impaired function which might, in some way, allow a sequence of events terminating in congestive cardiac failure. ${ }^{12}$ We have previously reported a quantitative study of $T$ cell populations in three groups: eight patients with congestive cardiomyopathy, eight patients with heart failure caused by coronary disease, and eight normal controls. ${ }^{3}$ There was no significant difference in the ratio of helper/suppressor cells between the groups, although there was a tendency for both heart failure groups to have higher ratios. This was due to both an increase in helper cells and a decrease in suppressor cells in both patient groups, but none of these differences was significant. These results suggested to us that any defect of suppressor cells must lie in abnormal function rather than in reduced numbers but we have failed to demonstrate a specific abnormality of suppressor cell function in the heart failure of congestive cardiomyopathy. We agree with Sanderson, however, that an immunological abnormality may be transient and detectable only in the early stage of congestive cardiomyopathy. All of our patients in both studies had been known to have congestive cardiomyopathy for more than three months.

P J Lowry, M D Gammage, R A Thompson, W A Littler, Department of Cardiovascular Medicine, East Birmingham Hospital, Bordesley Green East, Birmingham B9 5ST.

\section{References}

1 Fowles RE, Bieber CP, Stinson EB. Defective in vitro suppressor cell function in idiopathic congestive cardiomyopathy. Circulation 1979;59:483-91.

2 Eckstein R, Mempel W, Bolte HD. Reduced suppressor cell activity in congestive cardiomyopathy and in myocarditis. Circulation 1982;65:1224-9.

3 Lowry PJ, Thompson RA, Littler WA. Cellular immunity in congestive cardiomyopathy: the normal cellular immune response. Br Heart J 1985;53:394-9.

\section{Notices}

\section{British Cardiac Society}

The Annual General Meeting for 1988 will take place in Belfast on 23 and 24 March 1988, and the closing date for receipt of abstracts was 4 January 1988.

\section{Cardiovascular intervention}

A workshop on coronary angioplasty and other related procedures will be held at the London Hospital on 28 and 29 April 1988. Inquiries to Dr M T Rothman, The London Hospital, London E1 1BB.

\section{Pacing and electrophysiology}

The annual scientific meeting of the North American Society of Pacing and Electrophysiology will take place in Los Angeles on 12 to 14 May 1988. Inquiries to NASPE, 13 Eaton Court, Wellesley Hills, MA 02181, USA. 